\title{
Preliminary study of abnormalities in saccade dynamics in patients with hyperthyroidism with no pre-existing eye damage
}

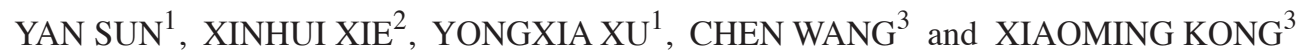 \\ ${ }^{1}$ Department of Geriatric Endocrinology, The First Affiliated Hospital of Anhui Medical University, Hefei, Anhui 230022; \\ ${ }^{2}$ Laboratory of Brain Stimulation and Biological Psychiatry, The Second People's Hospital of Huizhou, Huizhou, \\ Guangdong 516001; ${ }^{3}$ Department of Psychiatry, Anhui Mental Health Center, Hefei, Anhui 230022, P.R. China
}

Received April 27, 2019; Accepted December 13, 2019

DOI: $10.3892 /$ etm.2020.8461

\begin{abstract}
The present study investigated the relationship between hyperthyroidism and thyroid-associated ophthalmopathy by examining saccade dynamics to identify defects in eye tracking in patients with hyperthyroidism with no pre-existing eye damage and sensitive indicators that discriminated eye tracking ability in hyperthyroidism. A total of 33 outpatients with hyperthyroidism and 26 healthy controls participated in visually guided saccade (VGS) analysis. Patients with hyperthyroidism were divided into groups based on their medication status (medicated vs. unmedicated). Main sequence analysis was performed to identify differences in peak velocity and duration, and a general linear model (GLM) was used to identify differences in latency, peak acceleration and peak deceleration among the groups. The present study compared differences in the Spearman's correlation coefficient of the duration of saccades and the acceleration asymmetric index $\left(\mathrm{R}_{\mathrm{AD}}\right)$ among the groups. $\mathrm{V}_{\max }$ values $\left(\mathrm{V}_{\max }\right.$ was the asymptotic value of the PV of saccades of large amplitude) were significantly different between the healthy control and unmedicated-hyperthyroidism groups. The results of the GLM-based analysis indicated no significant differences in saccade latency among the three groups. Peak acceleration was significantly different between the healthy control and unmedicated-hyperthyroidism groups $(\mathrm{P}<0.01)$. Peak deceleration was significantly different between the healthy control, unmedicated- and medicated-hyperthyroidism groups $(\mathrm{P}<0.01) . \mathrm{R}_{\mathrm{AD}}$ was significantly different between the healthy control and medicated-hyperthyroidism groups $(\mathrm{P}=0.004)$. The results of the present study suggested that patients with hyperthyroidism with no pre-existing eye damage exhibited significantly altered saccade dynamics during VGS. Therefore,
\end{abstract}

Correspondence to: Dr Xiaoming Kong, Department of Psychiatry, Anhui Mental Health Center, 316 Huangshan Road, Hefei, Anhui 230022, P.R. China

E-mail:kxm186@163.com

Key words: hyperthyroidism, thyroid-associated ophthalmopathy, visually guided saccade, treatment
$\mathrm{R}_{\mathrm{AD}}$ may be used as an indicator to monitor the level of eye movement coordination.

\section{Introduction}

Thyroid-associated ophthalmopathy (TAO) is a complication of autoimmune thyroid disease (1). TAO is closely associated with hyperthyroidism (2). In total, $90 \%$ of TAO cases are caused by hyperthyroidism, but the relationships between TAO and the severity and duration of hyperthyroidism or thyroid function are unclear (3). In addition, the mechanisms underlying TAO pathogenesis and developmental processes are also unknown (4). As there is currently no effective therapy for TAO, the purpose of early treatment is to control immune inflammation, and later treatment aims to improve facial features and protect visual function (5). Corticosteroids are the main drugs used to control immune responses in the eye (6). However, corticosteroids are more effective when applied during the course of ocular inflammation symptoms in the early stage, and poorer effects are observed in patients with serious sequelae and chronic fibrosis (7). The methods used to evaluate TAO include ultrasonic B-scanning (8), CT (9) and MRI (10). However, TAO can only be diagnosed when morphological changes in the organization of the eye or eye muscles are observed (11). The cost of these inspection methods is high, and they are not suitable for use as early screening tools (12). Thus, the lack of sensitive objective examination tools for the early diagnosis of TAO remains an issue.

Saccades are a type of rapid eye movement pattern, during which the eyes move in the same direction insynchronized movements; this is an advanced feature of human eyes (13). TAO is a type of eye disease characterized by early pathological changes in eye organization and can cause hyperemia and edema or, in later stages, fibrosis in extra ocular muscles and orbital tissues that can lead to changes in eye tracking ability $(14,15)$. Therefore, checking the tracking ability of the eyes maybe useful for detecting early TAO.

Previous studies have investigated TAO and saccadic function, and the results have suggested that saccadic functions are abnormal in hyperthyroidism $(16,17)$. Our previous studies evaluated basic visually guided saccade (VGS) to explore the potential for eye damage in first-episode treatment-naive 
hyperthyreosis patients without pre-existing eye symptoms. The previous results demonstrated that the patients exhibited abnormal eye movements with increasing $\mathrm{V}_{\max }\left(\mathrm{V}_{\max }\right.$ was the asymptotic value of the PV of saccades of large amplitude) and peak acceleration and short saccade latency (18). The main sequence equation and general linear model (GLM) are considered to be reliable methods for analyzing eye movement data. To further explore the association between hyperthyroidism and $\mathrm{TAO}$, the present study used saccade tracking to evaluate the decline in eye tracking ability in patients with hyperthyroidism with no pre-existing eye damage. The present study investigated sensitive indicators of defects in eye tracking ability and factors related to abnormal eye tracking ability in hyperthyroidism.

\section{Materials and methods}

Participants. Patients with hyperthyroidism were randomly recruited from the endocrine clinic at the First Affiliated Hospital of Anhui Medical University from December 2014 to May 2015. Hyperthyroidism was diagnosed as serum triiodothyronine $\left(\mathrm{T}_{3}\right)$ and thyroxine $\left(\mathrm{T}_{4}\right)$ levels above the normal range in combination with lower thyroid-stimulating hormone (TSH) values. For each participant, serum hormone levels of $\mathrm{T}_{4}, \mathrm{~T}_{3}$ and TSH were measured in a chemiluminescence immunoassay performed at the Endocrinology Laboratory of The First Affiliated Hospital, Anhui Medical University. For $\mathrm{T}_{4}$, the normal range was $58.10-140.60 \mathrm{nmol} / \mathrm{l}$. For $\mathrm{T}_{3}$, the normal range was $0.92-2.79 \mathrm{nmol} / 1$. For TSH, the normal range was $0.550-4.780 \mathrm{IU} / \mathrm{ml}$. The present evaluation of eye symptoms was based on the standards developed for the NOSPECS system of classification of the American Thyroid Association (ATA) (19). The current accepted diagnostic criteria on TAO are based on standards developed by the NOSPECS classification; grade $>3$ can be diagnosed as TAO (20). In the present study, the NOSPECS in all patients was grade $\leq 3$. The healthy controls were recruited by advertisements in nearby districts and had no personal history of thyroid or other endocrine-related disease. None of the participants had myopia, astigmatism, other ametropia, epilepsy, mental retardation or a severe physical disease.

The participants were divided into a healthy control group $(n=26)$, an unmedicated-hyperthyroidism group $(n=15)$ and a medicated-hyperthyroidism group $(n=18)$. Treatment for the medicated-hyperthyroidism group included 5-30 mg methimazole or 100-300 mg prothiouracil and 10-30 mg propranolol hydrochloride. Smoking was also investigated; seven smoking cases were identified in the healthy control group, one case in the unmedicated-hyperthyroidism group and five cases in the unmedicated-hyperthyroidism group. In the present study, no women smoked, and no difference was observed between the numbers of smoking men in the three groups. Age and sex were similar among the three groups.

According to the Declaration of Helsinki, all participants enrolled in the study provided written detailed informed consent. Participation was voluntary, and patients were allowed to reject or withdraw at any point. The study was approved by the Ethics Committee of The First Affiliated Hospital of Anhui Medical University.
Procedures and stimulus. The experiment was created, performed and analyzed using Experiment Center Software 3.0 (SensoMotoric Instruments $\mathrm{GmbH}$ ). Each participant sat in a chair $\sim 60 \mathrm{~cm}$ in front of a liquid crystal display screen with their head fixed comfortably on a chinrest. An IVIEW X HISPEED eye tracker (SensoMotoric Instruments $\mathrm{GmbH}$ ) was used to record the participants' eye movements while they performed directed tasks. The sampling rate was set to1, $250 \mathrm{~Hz}$, with the accuracy rate $<1^{\circ}$ and a spatial resolution of $\sim 0.01^{\circ}$. Patients underwent VGS, in which a single black dot $\sim 1^{\circ}$ in sight was randomly displayed on a gray background. The position of the dot was randomized, and the duration was also randomized between 1,000-1,500 ms with no intervals between dots. The participants were asked to keep visually fixated on the black dots, and saccades were guided by the changing positions of the dots. A total of 10 practice and 100 formal trials were performed; the practice trials were not included in the analyses. Saccade duration was detected with a velocity threshold of $40 \%$ s and was recorded automatically by computer. The following parameters were recorded during each saccade: i) amplitude; ii) duration; iii) latency; iv) peak velocity (PV); v) peak acceleration; and vi) peak deceleration.

Statistical analysis. All statistical analyses were performed using SPSS 20.0 (IBM Corp.). The statistical methods included the $\chi 2$ test, GLM analysis, Bonferroni's correction test, the Kruskal-Wallis test and Dunn's test.

Main sequence analysis was performed by fitting saccade PV and amplitude in the following main sequence equation (equation 1): Peak Velocity $=\mathrm{V}_{\max } \times\left(1-\mathrm{e}^{- \text {Amplitude/c }}\right)$, where $\mathrm{V}_{\max }$ was the asymptotic value of the PV of saccades of large amplitude, and c was the amplitude constant. A nonlinear regression procedure was used on all three groups to estimate $\mathrm{V}_{\max }$ and the constant $\mathrm{c}$ to fit the main sequence.

A GLM was used to determine the differences in latency, peak acceleration and peak deceleration among the groups. Saccade latency, peak acceleration and peak deceleration were set as the dependent variables, where as PV, duration and amplitude were used as covariates. Pairwise comparisons between groups were performed using the Bonferroni correction.

The individual saccade data processing method was performed based on a previous study by Cui et al (21). Each saccade can be used to calculate the acceleration asymmetric index (AAI) using equation 2. As acceleration (or deceleration) represents the rate of changes in velocity, the reciprocal of the velocity curve (the curve slope), AAI, reflects the asymmetry of the curve in terms of the slope of the velocity curve. Equation 2: AAI = (|Acceleration peak| $-\mid$ Deceleration peakl $) /(\mid$ Acceleration peakl + IDeceleration peakl).

Further statistical analysis of the saccade data was performed using the SPSS 20.0 statistical package (IBM Corp.). The Spearman's correlation coefficient of the duration of saccades and the AAI $\left(R_{A D}\right)$ was calculated. The $R_{A D}$ and corresponding P-value were calculated for each of the subjects. $R_{A D}$ reflects the coordination of the saccade as the relationship between the asymmetry of acceleration and the duration of the saccade (21). $\mathrm{P}<0.05$ was considered to indicate a statistically significant difference. 
Table I. Basic information of the three groups.

\begin{tabular}{|c|c|c|c|c|}
\hline Parameter & HC group $(n=26)$ & U-H group $(n=15)$ & M-H group (n=18) & P-value \\
\hline Sex, male:female & $12: 14$ & $2: 13$ & $7: 11$ & 0.105 \\
\hline Age, years & $36.5 \pm 8.4$ & $39.2 \pm 12.7$ & $41.4 \pm 13.0$ & 0.346 \\
\hline No. of smoking men & 7 & 1 & 5 & 0.172 \\
\hline Disease course, months & n.a. & $11.4 \pm 18.4$ & $32.3 \pm 82.6$ & 0.109 \\
\hline
\end{tabular}

HC, healthy control;U-H, unmedicated-hyperthyroidism; M-H, medicated-hyperthyroidism.

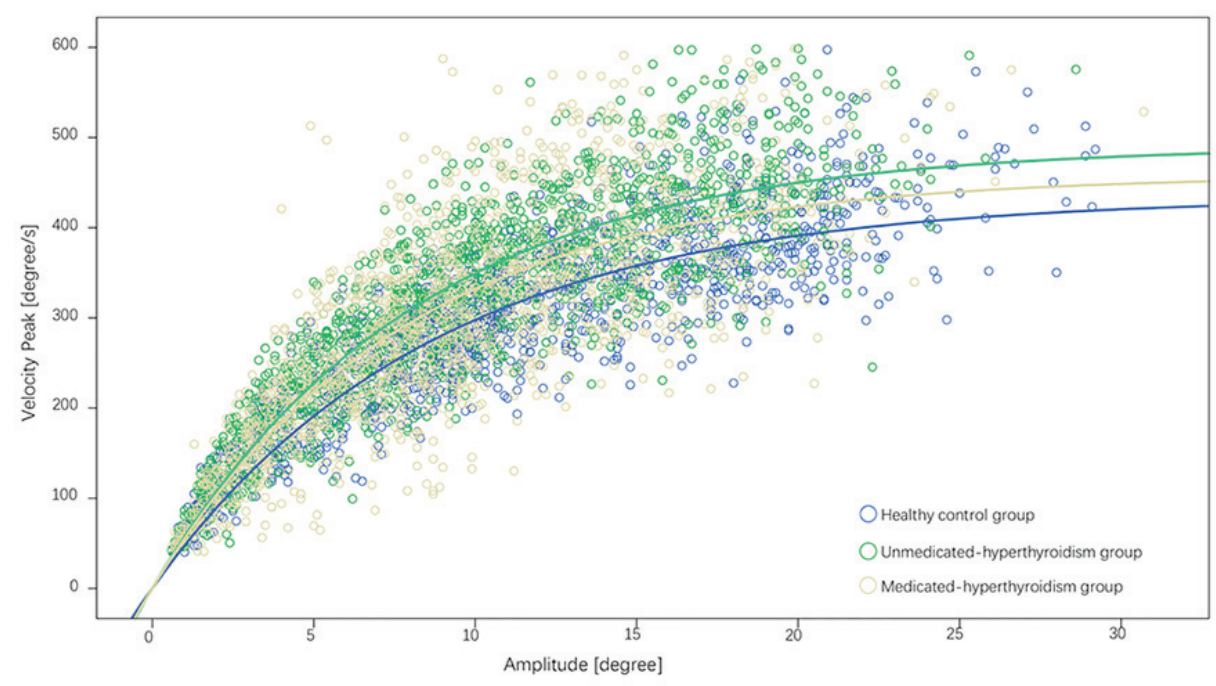

Figure 1. Typical saccadic main sequences for amplitude and peak velocity of the three groups. Curve of peak velocity.

\section{Results}

Patient data. The basic information of the three groups is presented in Table I. The patient age ranged from 22-52 years in the healthy control group, and 25-66 and 19-60 years in the unmedicated- and medicated-hyperthyroidism groups, respectively. No significant differences were observed in patient age and sex.

Typical saccadic main sequences for amplitude and peak velocity of the three groups. Using the main sequence equation, the results of the present study indicated that the $\mathrm{V}_{\max }$ values were $443.859^{\circ}$ s in the healthy control group $(95 \% \mathrm{CI}$, $433.719-453.999)$ and $490.736 \%$ (95\% CI, 478.656-502.817) and $458.159 \%$ s (95\% CI, 443.169-473.150) in the unmedicatedand medicated-hyperthyroidism groups, respectively. The $\mathrm{V}_{\max }$ values were significantly different between the healthy control and unmedicated-hyperthyroidism groups, but were not significantly different between the healthy control and medicated-hyperthyroidism groups. The fitting curves of the three groups are presented in Fig. 1.

Saccades. The results of the GLM-based analysis performed using an amplitude of $10.155^{\circ}$, a PV value of $300.996 \%$ and a duration of $60.06 \mathrm{~ms}$ indicated that saccade latencies were $238.587 \mathrm{~ms}$ in the healthy control group (95\% CI, 230.792-246.382), $237.437 \mathrm{~ms}$ in the unmedicated-hyperthyroidism group
(95\% CI, 228.608-246.267) and $240.716 \mathrm{~ms}$ in the medicated-hyperthyroidism group (95\% CI, 233.168-248.263). No significant differences were observed in saccade latency between the three groups. The peak acceleration of the three groups was $13,690.682^{\circ} / \mathrm{s}^{2}(95 \% \mathrm{CI}, 13,610.496-13,770.868)$, $13,848.965 \% \mathrm{~s}^{2}(95 \% \mathrm{CI}, 13,758.137-13,939.794)$ and $13,741.763^{\circ} / \mathrm{s}^{2}$ (95\% CI, 13,664.121-13,819.406), respectively. Peak acceleration was significantly different between the healthy control and unmedicated-hyperthyroidism groups $(\mathrm{P}=0.01)$. The peak decelerations were $-10,084.399 \% \mathrm{~s}^{2}$ (95\% CI, -10,207.519- -9961.278), $\left.-10,480.185^{\circ} / \mathrm{s}^{2}(95 \% \mathrm{CI},-10,619.647--10,340.724)\right]$ and $-10,317.593 \% \mathrm{~s}^{2}$ (95\% CI, -10,436.808- -10,198.377), respectively. Pairwise comparisons between groups were performed using the Bonferroni correction. Significant differences were identified between the healthy control and unmedicated-hyperthyroidism groups and between the healthy control and medicated-hyperthyroidism groups $(\mathrm{P}<0.01)$, but no significant differences were present between the two hyperthyroidism groups. The results of the saccade analysis are presentedin Table II.

$R_{A D}$ analysis. Each saccade was used to calculate an AAI, and the $\mathrm{R}_{\mathrm{AD}}$ of every participant was calculated. A Kruskal-Wallis test revealed that the difference of $\mathrm{R}_{\mathrm{AD}}$ among the three groups was significant $(\mathrm{P}=0.013)$. Dunn's post hoc test demonstrated that $R_{A D}$ was significantly different between the healthy control and medicated-hyperthyroidism groups $(\mathrm{P}=0.004$; Fig. 2), but no significant differences were identified between 
Table II. The general descriptions of saccades.

\begin{tabular}{lccc}
\hline Parameter & HC group & U-H group & M-H group \\
\hline Saccade latency, ms & $238.587 \pm 3.976$ & $237.437 \pm 4.504$ & $240.716 \pm 3.850$ \\
Peak acceleration, $\% \mathrm{~s}^{2}$ & $13,690.682 \pm 40.901$ & $13,848.965 \pm 46.329^{\mathrm{a}}$ & $13,741.763 \pm 39.603$ \\
Peak deceleration, $^{\circ} / \mathrm{s}^{2}$ & $-10,084.399 \pm 62.801$ & $-10,480.185 \pm 71.136^{\mathrm{a}}$ & $-10,317.593 \pm 60.809^{\mathrm{a}}$ \\
\hline
\end{tabular}

${ }^{\mathrm{a}} \mathrm{P}<0.05$ vs. the healthy control group. Data from the general linear model analysis. Data are presented as the mean $\pm \mathrm{SD}$. HC, healthy control; U-H, unmedicated-hyperthyroidism; M-H, medicated-hyperthyroidism.

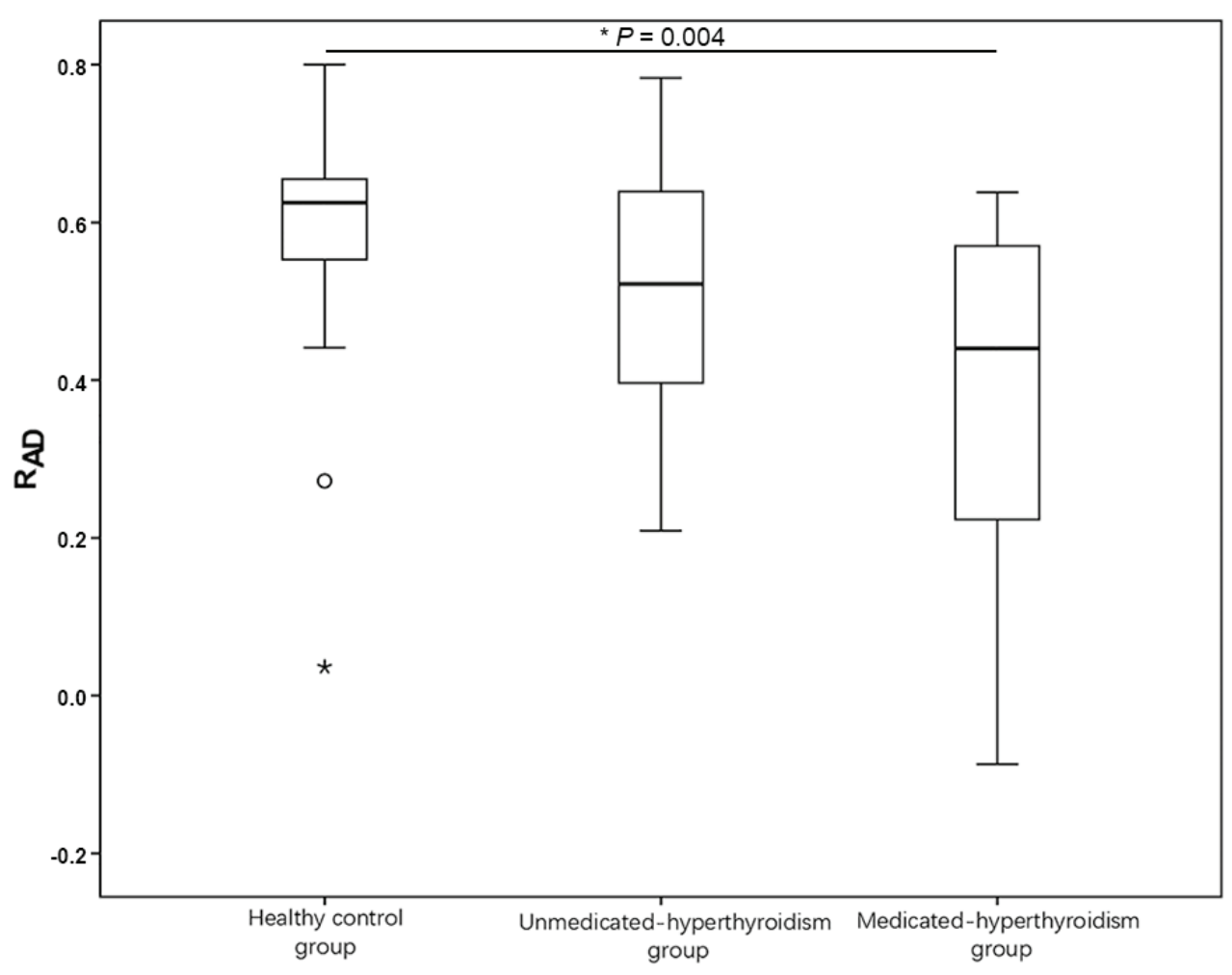

Figure 2. RAD values of the three groups. ${ }^{*}<0.05$. RAD, Spearman's correlation coefficient of the duration of saccades and the acceleration asymmetric index.

the healthy control and unmedicated-hyperthyroidism groups $(\mathrm{P}=0.283)$ or between the two hyperthyroidism groups $(\mathrm{P}=0.056)$.

\section{Discussion}

Eye-tracking is a technique that monitors the ability of the eyes to move in a coordinated manner (22). Eye movement processes require central generators, nerve conduction systems and eye movement systems. These processes are reflected in technologies that are widely used in industry, mechanical manufacturing and human engineering (23). In medicine, eye-tracking systems are mainly applied in neuropsychology and ophthalmology research (24). In the fields of psychology and psychiatry, eye-tracking technology has recently been confirmed to be useful in diagnosing various diseases, including Parkinson's disease (25), Huntington's (26), abnormal gaze (27), nervous system degenerative diseases (28) and early Alzheimer's disease (29). Home eye-tracking systems can be used to follow up patients with vertigo (30).
Another previous study also demonstrated that patients with schizophrenia exhibit significant saccadic changes (21). There is an increasing interest in eye-tracking technologies due to their convenience and objectivity.

In the development of TAO, the pathological changes in the eye only present as tissue edema in the early stages (31). During this period, eye damage is minor and not easily detected by examinations such as B-ultrasound and CT (11). Orbital tissue fibrosis develops between one and several years (4). Once fibrosis occurs, the organization of the eyeball cannot be restored to its former healthy status, and the patient still experiences residual symptoms and chronic external eye muscle dysfunction (4). During this period, the damage to saccades in patients is obvious, which is the consensus reached by the majority of TAO studies related to saccades $(15,16)$. It is difficult to treat TAO once fibroplasia occurs (32). Therefore, there is a need for a diagnostic technique to detect the early inflammatory stage of TAO. The current diagnosis of TAO is based on standards developed by the NOSPECS classification of the ATA. A grade $>3$, which is based on the patient's exophthalmia 
level, TAO can be diagnosed (19). Lower NOSPECS grading (0-2) status is based primarily on eyeball symptoms and signs, which can be easily missed by doctors (16). At present, the lack of sensitive objective examinations for patients with early TAO remains problematic (33). In the present study, patients with hyperthyroidism had NOSPECS grade $\leq 3$. The objective of the present study was to identify a cost-effective and simple method to evaluate early ocular abnormalities in patients with hyperthyroidism.

Our previous study analyzed the saccades of patients with hyperthyroidism without obvious eye symptoms and revealed a significant difference in saccade dynamics, including $\mathrm{V}_{\max }$, increased peak acceleration and short latencies, during VGS between affected patients and healthy controls (18). These previous results were similar to those published in other previous studies (16).

The present study expanded the sample size and divided the hyperthyroidism groups based on their medication status. The purpose of dividing the patients into three groups in this study was to systematically research the changes in eye movements of hyperthyroidism during treatment. In clinical practice, a number of patients do not experience improvement in ocular symptoms, even when aggravated gradually with the control of hyperthyroidism. These patients may be the high-risk groups of TAO (34). However, there is no effective method to predict these high-risk patients. Therefore, the present study investigated patients with hyperthyroidism receiving anti hyperthyroidism drugs to understand the status of eye movements during the treatment process. The results of the present study indicated that patients in the unmedicated-hyperthyroidism group exhibited the highest $\mathrm{V}_{\max }$ and peak acceleration, which was significantly different compared with the healthy control group. The results were similar to those of our previous study (18). The unmedicated-hyperthyroidism group included patients with a first episode and those with recurrent hyperthyroidism in whom the symptoms of hyperthyroidism were obvious. The reasons for abnormal eye movements have been associated with an increase in sympathetic nerve excitability (35). Otherwise, the regulation of saccadic central generator mechanisms has not yet been extensively elucidated $(36,37)$. The present results suggested that the $\mathrm{V}_{\max }$ and peak acceleration were increased in the medicated-hyperthyroidism group, but were not significantly different compared with the healthy control group. The present study hypothesized that this effect was related to the use of anti hyperthyroidism drugs and $\beta$-blockers in the medicated-hyperthyroidism group. The prescription of $\beta$-blockers in patients with hyperthyroidism can improve symptoms associated with sympathetic activation and the basal metabolic rate, which may be the reason for the declines in $\mathrm{V}_{\max }$ and peak acceleration observed in hyperthyroidism (38). The relatively long duration of the disease course in the medicated-hyperthyroidism group could be due to another reason; the courses of hyperthyroidism were $11.4 \pm 18.4$ months in the unmedicated-hyperthyroidism group and $32.3 \pm 82.6$ months in the medicated-hyperthyroidism group; the difference was not significant since there were fewer participants in the two hyperthyroidism groups and higher standard deviations in the medicated-hyperthyroidism group. The adaptability of the central neural saccade generator gradually changes over time (39). Peak deceleration was significantly higher in hyperthyroidism groups compared with the healthy control group, indicating that eye movement velocity declined faster during the deceleration period in the hyperthyroidism group. Baloh et al (40) have demonstrated that saccades do not change constantly, implying that acceleration is faster compared with deceleration, and this biases changes in the variation of the saccade amplitude. In the present study, peak acceleration and deceleration were significantly higher in the unmedicated-hyperthyroidism group compared with the healthy control group, and their eye movement curves were steep, indicating that the eye movement processes sped up and slowed down faster. In the medicated-hyperthyroidism group, only a rapid decline was observed in eye movement, and the mechanism underlying this effect is not yet known.

The present study used $\mathrm{R}_{\mathrm{AD}}$ to characterize the eye movements of patients with hyperthyroidism. The $\mathrm{R}_{\mathrm{AD}}$ was first suggested by Cui et al (21) and has since then been elaborated by Xie Xinhui et al (41), who identified a number of indicators that describe the dynamic characteristics of the eye, such as $R_{A A}\left(R_{A A}\right.$ is the Spearman's correlation coefficient of the amplitude of saccades and the AAI). The saccadic curve is asymmetric, and the slope of the velocity curve reflects changes in the trends of acceleration and deceleration (40). Based on this characteristic, the asymmetry of the curve is calculated from the angle of the slope of the velocity curve, which is represented by the AAI. The correlation between the AAI and the duration of saccades can be used as an indicator of how well eye movements are coordinated (21).

In the present study, $\mathrm{R}_{\mathrm{AD}}$ was significantly different between the healthy control and medicated-hyperthyroidism groups, and this result reflected the finding that saccadic coordination was poor in the medicated-hyperthyroidism group. There was no significant difference between the healthy control and unmedicated-hyperthyroidism groups. The present study hypothesized that anti hyperthyroidism drugs decreased the excitability of the sympathetic system and influenced saccadic coordination. As the duration of the medicated-hyperthyroidism group was relatively long, the process of ocular inflammation and its underlying pathology may have led to changes in saccadic coordination. The disease course of TAO follows the Rundle curve: It enters an early inflammatory progression stage followed by a platform phase prior to gradually moving into a low phase. As TAO progresses, chronic fibrosis in eye tissues can decrease eye coordination (42). Previous studies have revealed that the saccades of patients with a long TAO course were abnormal (16); however, to the best of our knowledge, no study has investigated TAO with the disease course of hyperthyroidism. In future studies, the authors of the present study will continue to analyze the relationship between the course of hyperthyroidism and the initiation of TAO pathogenesis.

Considering the characteristics of exploratory research, the present study used a small sample size. Due to the high accuracy and small heterogeneity of eye movement research, the sample size of eye movement studies generally smaller compared with other clinical researches (16-18). According to previous results in similar eye movement studies $(16,17)$, each group had $\sim 10$ cases, which met the statistical requirements of saccade analysis. Therefore, the present study used the current sample size in this exploratory study. In the future, increased case numbers will be used for further investigation. 
The present study was an explanatory study and funding was limited; as a result, the imaging examinations, including B-scanning, CT and MRI were not performed. In future work, the authors will improve the test design and include imaging methods to increase understanding of the association between eye morphology changes and eye movement.

Previous studies have reported that TAO is an organ-specific autoimmune disease, and common antigens (are present in the thyroid and eye (43). A variety of auto-antibodies against auto-antigens have been identified in patients with TAO, such as thyroglobulin, thyroid peroxidase and TSH receptor antibodies (44). TSH receptor also exists in the orbital tissues and extraorbital muscles of patients with TAO; therefore, the TSH receptor may be associated with $\mathrm{TAO}$, although this theory has not been fully investigated (45). The present study is a preliminary exploratory study and did not collect complete antibody data. Future experiments will focus on the effect of antibodies on TAO.

The results of the present exploratory study suggested that patients with hyperthyroidism without obvious eye symptoms exhibited a significant difference in the dynamics of saccades during VGS compared with healthy controls. The coordination of eye movements in patients treated with anti-hyperthyroidism medications was not improved, which may be related to the progressive prolongation of the course of hyperthyroidism. $R_{A D}$ maybe used as an indicator to monitor the level of eye movement coordination. Saccade tracking examination may have potential value for the early detection of TAO and requires further studies in patients with hyperthyroidism.

\section{Acknowledgements}

Not applicable.

\section{Funding}

The present study was supported by The University Natural Science Research Project of Anhui Province (grant no. KJ2018A0196), The Applied Medicine Research Project of the Hefei Health and Family Planning Commission (grant no. hwk2017yb015) and The Doctoral Foundation of the First Affiliated Hospital of Anhui Medical University (grant no. 2017-1280).

\section{Availability of data and materials}

All data generated or analyzed during the present study are included in this published article.

\section{Authors' contributions}

YS and XMK designed and performed the research. XHX and CW performed the saccade experiment. YS and YXX collected the data from the participants. XHX and YS analyzed the data and wrote the final article.

\section{Ethics approval and consent to participate}

The study was approved by the Ethics Committee of The First Affiliated Hospital of Anhui Medical University, and informed consent was provided by the patients and/or guardians.

\section{Patient consent for publication}

Not applicable.

\section{Competing interests}

The authors declare that they have no competing interests.

\section{References}

1. Joffe B, Gunji K, Panz V, Zouvanis M, Swanson J, Ackrell BA and Wall JR: Thyroid-associated ophthalmopathy in black South African patients with Graves' disease: Relationship to antiflavoprotein antibodies. Thyroid 8: 1023-1027. 1998.

2. Prummel MF, Wiersinga WM, Mourits MP, Koornneef L, Berghout A and van der Gaag R: Effect of abnormal thyroid function on the severity of Graves' ophthalmopathy. Arch Intern Med 150: 1098-1101, 1990.

3. Brent GA: Clinical practice. Graves' disease. N Engl J Med 358: 2594-2605, 2008.

4. Teissier MP and Lopez S: Thyroid-associated ophthalmopathy: Physiopathology, endocrine status. J Fr Ophtalmol 27: 806-809, 2004 (In French).

5. Kahaly GJ, Hardt J, Petrak F and Egle UT: Psychosocial factors in subjects with thyroid-associated ophthalmopathy. Thyroid 12: 237-239, 2002.

6. Hart RH, Kendall-Taylor P, Crombie A and Perros P: Early response to intravenous glucocorticoids for severe thyroid-associated ophthalmopathy predicts treatment outcome. J Ocul Pharmacol Ther 21: 328-336, 2005.

7. Wiersinga WM: Management of Graves' ophthalmopathy. Nat Clin Pract Endocrinol M 3: 396-404, 2007.

8. Rajabi MT, Papageorgiou K, Taban M, Hwang CJ, Hosseini SS, Rajabi MB and Goldberg RA: Ultrasonographic motion analysis of lower eyelid compartments in patients with chronic thyroid associated ophthalmopathy. J Curr Ophthalmol 29: 310-317, 2017.

9. Fang ZJ, Zhang JY and He WM: CT features of exophthalmos in Chinese subjects with thyroid-associated ophthalmopathy. Int J Ophthalmol 6: 146-149, 2013.

10. Shen J, Jiang W, Luo Y, Cai Q, Li Z, Chen Z, Hu S and Tang L: Establishment of magnetic resonance imaging $3 \mathrm{D}$ reconstruction technology of orbital soft tissue and its preliminary application in patients with thyroid-associated ophthalmopathy. Clin Endorinol (oxf) 88: 637-644, 2018 .

11. Stan MN, Garrity JA and Bahn RS: The evaluation and treatment of graves ophthalmopathy. Med Clin North Am 96: 311-328, 2012.

12. Gould DJ, Roth FS and Soparkar CN: The diagnosis and treatment of thyroid-associated ophthalmopathy. Aesthetic Plast Surg 36: 638-648, 2012.

13. McDowell JE, Dyckman KA, Austin BP and Clementz BA. Neurophysiology and neuroanatomy of reflexive and volitional saccades: evidence from studies of humans. Brain Cogn 68:255$270,2008$.

14. Feldon SE and Unsöld R: Graves' ophthalmopathy evaluated by infrared eye-movement recordings. Arch Ophtalmol 100: 324-328, 1982.

15. Feldon SE, Levin L and Liu SK: Graves' ophthalmopathy. Correlation of saccadic eye movements with age, presence of optic neuropathy and extra ocular muscle volume. Arch Ophthalmol 108: 1568-1571, 1990.

16. Schworm HD, Bolzani R, Benassi M, Tallstedt L, Rydberg A, Lennerstrand $G$ and Ygge J: Changes of saccadic eye movements in thyroid-associated ophthalmopathy. Acta Ophthalmol 90: 713-720, 2012.

17. Schworm HD, Heufelder AE, Kunze A, Welge E and Boergen KP: Clinical significance of saccade analysis in early active Graves' ophthalmopathy. Invest Ophthalmol Vis Sci 41: 1710-1718, 2000.

18. Sun Y, Kong X, Wang C, Xu Y, Wang K and Zhu D: Abnormalities in saccade dynamics in first-episode treatment-naive hyperthyreosis patients with no pre-existing eye damage: A primary exploratory study. Int J ClinExp Med 9:11626-11632, 2016.

19. Van Dyk HJ: Orbital Graves' disease. A modification of the 'NO SPECS' classification. Ophthalmology 88: 479-483, 1981.

20. Dolman PJ: Evaluating Graves' orbitopathy. Best Pract Res Clin Endocrinol Metab 26: 229-248, 2012. 
21. Cui H, Liu XH, Wang KY, Zhu CY, Wang $\mathrm{C}$ and Xie $\mathrm{XH}$ Association of saccade duration and saccade acceleration/deceleration asymmetry during visually guided saccade in schizophrenia patients. PLoS One 9: e97308, 2014.

22. Ferhat $O$ and Vilariño F: Low cost eye tracking: The current panorama. Comput Intell Neurosci 2016: 8680541, 2016.

23. Guo S, Pan S, Shi L, Guo P, He Y and Tang K: Visual detection and tracking system for a spherical amphibious robot. Sensors (Basel) 17: pii: E870, 2017.

24. Asan O and Yang Y: Using eye trackers for usability evaluation of health information technology: A systematic literature review. JMIR Hum Factors 2: e5, 2015.

25. Srivastava A, Sharma R, Sood SK, Shukla G, Goyal V and Behari M: Saccadic eye movements in Parkinson's disease. Indian J Ophthalmol 62: 538-544, 2014

26. Turner TH, Goldstein J, Hamilton JM, Jacobson M, Pirogovsky E, Peavy $\mathrm{G}$ and Corey-Bloom J: Behavioral measures of saccade latency and inhibition in manifest and premanifest Huntington's disease. J Mot Behav 43: 295-302, 2011.

27. Vinny PW and Lal V: Gaze disorders: A clinical approach. Neurol India 64: 121-128, 2016.

28. MacAskill MR and Anderson TJ: Eye movements in neurodegenerative diseases. Curr Opin Neurol 29: 61-68, 2016.

29. Fernández G,Manes F,Politi LE, Orozco D, Schumacher M,Castro L, Agamennoni $\mathrm{O}$ and Rotstein NP: Patients with mild Alzheimer's disease fail when using their working memory: Evidence from the eyetracking technique. J Alzheimers Dis 50: 827-838, 2016.

30. Termsarasab P, Thammongkolchai T, Rucker JC and Frucht SJ: The diagnostic value of saccades in movement disorder patients: A practical guide and review. J Clin Mov Disord 2: 14, 2015.

31. Wang L and Ma JM: Progression of the pathogenesis of thyroid associated ophthalmopathy. Zhonghua Yan Ke Za Zhi 53: 474-480, 2017 (In Chinese).

32. Madaschi S, Rossini A, Formenti I, Lampasona V, Marzoli SB, Cammarata G, Politi LS, Martinelli V, Bazzigaluppi E, Scavini M, et al: Treatment of thyroid-associated orbitopathy with rituximab-a novel therapy for an old disease: Case report and literature review. Endocr Pract 16: 677-685, 2010.

33. Savku E and Gündüz K: Diagnosis, follow-up and treatment results in thyroid ophthalmopathy. Turk J Ophthalmol 45: 156-163, 2015.
34. Bartalena L, Marcocci C and Pinchera A: Treating severe Graves' ophthalmopathy. Baillieres Clin Endocrinol Metab 11: 521-536, 1997.

35. Tani J, Gopinath B, Nguyen B and Wall JR: Extraocular muscle autoimmunity and orbital fat inflammation in thyroid-associated ophthalmopathy. Expert Rev Clin Immunol 3: 299-311, 2007.

36. Kommerell G, Olivier D and Theopold H: Adaptive programming of phasic and toniccomponents in saccadic eye movements. Investigations of patients with abducens palsy. Invest Ophthalmol 15: 657-660, 1976.

37. Inchingolo $\mathrm{P}$, Accardo A, Da Pozzo S, Pensiero S and Perissutti P: Cyclopean and disconjugate adaptive recovery from post-saccadic drift in strabismic children before and after surgery. Vision Res 36: 2897-2913, 1996.

38. De Leo S, Lee SY and Braverman LE: Hyperthyroidism. Lancet 388: 906-918, 2016.

39. Straube A, Fuchs AF, Usher S and Robinson FR: Characteristics of saccadic gain adaptationin rhesus macaques. J Neurophysiol 77: 874-895, 1997.

40. Baloh RW, Sills AW, Kumley WE and Honrubia V: Quantitative measurement of saccade amplitude, duration, and velocity. Neurology 25: 1065-1070, 1975.

41. Xie Xinhui, Liu Xiaohui, Wang Chen, Zhu Chunyan, Cui Hong and Wang Keyong: Lower correlation between saccade velocity asymmetry and amplitude during visually guided saccade in schizophrenia patients. Chin J Psychiatry 47:276-280,2014.

42. Rundle FF: Eye signs of Graves' disease. Thyroid 171-197, 1964.

43. Naik V, Khadavi N, Naik MN, Hwang C, Goldberg RA, Tsirbas A, Smith TJ and Douglas RS: Biologic therapeutics in thyroid-associated ophthalmopathy: Translating disease mechanism into therapy. Thyroid 18: 967-971, 2008.

44. Kaspar M, Archibald C, De BA, Li AW, Yamada M, Chang CH, Kahaly G and Wall JR: Eye muscle antibodies and subtype of thyroid-associated ophthalmopathy. Thyroid 12: 187-191, 2002.

45. Bahn RS: Graves' ophthalmopathy. N Engl J Med 362: 726-738, 2010. International (CC BY-NC-ND 4.0) License. 